\title{
A triagem auditiva neonatal na Rede Municipal do Rio de Janeiro, Brasil
}

\author{
Newborn hearing screening \\ in Rio de Janeiro's municipal network, Brazil
}

Priscila Tavares Lima ${ }^{1}$

Márcia Goldfeld Goldbach ${ }^{2}$

Márcia Cavadas Monteiro ${ }^{2}$

Márcia Gonçalves Ribeiro ${ }^{3}$

${ }^{1}$ Hospital Universitário Clementino Fraga Filho, Universidade Federal do Rio de Janeiro (UFRJ). R. Rodolpho Paulo Rocco 255, Cidade Universitária. 21941-913 Rio de Janeiro RJ

Brasil.priscilalima@ufrj.br ${ }^{2}$ Departamento de Fonoaudiologia, Faculdade de Medicina, UFRJ.

${ }^{3}$ Departamento de Pediatria, Faculdade de Medicina, UFRJ.
Abstract Hearing deficiencies are a prevalent disease and justify the need for regulation of the Laws and their execution through Hearing Health Care Ordinances. In line with public policies, maternity hospitals that were part of the network began to implement the Newborn Hearing Screening (NHS) service, as had occurred in the city of Rio de Janeiro. The otoacoustic emissions test is used for NHS as it is a rapid and highly reliable method that is easy to perform and gives objective results. The scope of this article is to get fully acquainted with the assistance and care for the hearing health of newborns in maternity wards of the Municipal Health Grid. It is an observational, descriptive, cross-sectional analysis with frequency distribution, and was conducted at SMS-RJ Maternity hospitals that perform NHS. Three maternity hospitals with NHS ( $A, B$ and $C)$ were identified, in which 1,865 live newborns were recorded. Of this total, $40.5 \%$ performed the NHS exam. In maternity hospitals $A$ and $B$, the NHS exam was applied to $54.6 \%$, of which $97.3 \%$ passed and only $1.8 \%$ failed and needed to be referred to the high complexity unit. The NHS is the initial stage of the Hearing Health Care Program for the newborn. It is important that the NHS services should be fully integrated into the network through the Hearing Health Care Program.

Key words Deafness, Hearing screening, Hearing
Resumo A deficiência auditiva é doença prevalente e justifica a necessidade de regulamentação das Leis e suas execuções através das Portarias de Atenção à Saúde Auditiva. Diante das politicas públicas, as maternidades que estavam inseridas na rede iniciaram processo de implantação do serviço de Triagem Auditiva Neonatal (TAN), como ocorrido Município do Rio de Janeiro. Para TAN utiliza-se o exame de Emissões Otoacústicas por ser um método rápido, de alta fidedignidade, fácil execução, com resultados objetivos. O objetivo deste artigo é conhecer melhor a assistência e o cuidado à saúde auditiva dos RN nas maternidades da Rede Municipal de Saúde. Estudo observacional, descritivo, transversal, análise descritiva com distribuição de frequências, e teve como objeto as Maternidades da Secretaria Municipal de Saúde-RJ que realizavam TAN. Foram identificadas três maternidades com TAN ( $A, B$ e $C$ ). Nelas foram registrados $1.865 R N$ vivos. Destes, $o$ total que realizou triagem foi de 40,5\%. Nas maternidades A e B, 54,6\% realizaram TAN e destes, 97,3\% passaram na TAN e apenas 1,7\% falharam e precisariam ser encaminhados para o serviço de alta complexidade. A TAN é o início do Programa de Atenção a Saúde Auditiva do RN. É importante que os serviços de TAN funcionem integrados à rede através do Programa de Atenção à Saúde Auditiva.

Palavras-chave Surdez, Triagem auditiva, Audição 


\section{Introdução}

A deficiência auditiva é doença prevalente na população brasileira e justifica a necessidade de regulamentação das Leis Federal e Municipal e suas execuções através das Portarias de Atenção à Saúde Auditiva no 2073/GM e no 587 de 2004; sua incidência é de 1 a 3:1000 recém-nascidos (RN) vivos em alojamento conjunto e de 2 a 4:100 para os RN que ficam em Unidade de Terapia Intensiva Neonatal (UTIN) $)^{1,2}$, considerada relativamente alta dentre as doenças triadas ao nascimento, sendo 100 vezes mais frequente que a Fenilcetonúria e 10 vezes mais que o Hipotireoidismo Congênito ${ }^{3,4}$. Quando a deficiência auditiva é diagnosticada e tratada precocemente, gera redução dos custos em saúde pública e educação, uma vez que há possibilidade de desenvolvimento linguístico, educacional e de inclusão social. Assim, o desenvolvimento das crianças com deficiência auditiva pode ficar próximo ao das crianças ouvintes da mesma faixa etária ${ }^{5-7}$. O Comitê Brasileiro sobre Perdas Auditivas na Infância ${ }^{8}$ reafirma que o diagnóstico da deficiência auditiva deve ser realizado até os três meses de idade e a intervenção iniciada até os seis meses, recomendação semelhante internacionalmente ${ }^{9}$.

Diante das políticas públicas em Saúde Auditiva, as maternidades que estavam inseridas na rede e referenciavam pacientes para os serviços de alta complexidade, iniciaram processo de implantação do serviço de Triagem Auditiva, como foi o caso do Município do Rio de Janeiro, que desde 2000 tem regulamentada a Lei do "Teste da orelhinha" no território municipal ${ }^{10}$. Esta lei ganhou reforço com a obrigatoriedade desta avaliação em território nacional através da Lei Federal $^{11}$.

A utilização das Emissões Otoacústicas na Triagem Auditiva Neonatal (TAN) revela-se vantajosa por ser um método rápido, de alta fidedignidade, fácil execução, com resultados objetivos e que pode ser realizado no bebê em sono natural $^{12}$. Para realização da TAN utiliza-se a pesquisa das emissões otoacústicas (EOA) que avaliam a integridade das células ciliadas externas (CCE) encontradas na cóclea, responsáveis pela amplificação do som. A presença das EOA indica bom funcionamento coclear (CCE) e a ausência indica a necessidade de avaliações complementares para investigação de possível perda auditiva. Sendo assim, no modo triagem consideramos que o recém-nascido "passou", quando obteve respostas dentro dos critérios de normalidade das EOA transiente para TAN, que são: reprodutibilidade geral $\geq 50 \%$ e estabilidade da sonda e reprodutibilidade das bandas de frequência $\geq 70 \%$ e amplitude das respostas por banda de frequência $\geq$ 6 em $2.4,3.2$ e $4 \mathrm{kHz}^{13}$. Quando o recém-nascido não atinge os critérios de normalidade, é dito que "falhou"14. De forma complementar, o Comitê Brasileiro de Perdas Auditivas na Infância sugere a pesquisa do reflexo cócleo-palpebral ${ }^{15}$.

A proporção da Deficiência Auditiva na população brasileira, que segundo o último senso corresponde a $16,7 \%{ }^{16}$, e suas consequências nos âmbitos social e econômico, foram um dos motivadores para a implementação da Política Nacional de Atenção à Saúde Auditiva pelo Ministério da Saúde. A Portaria GM 2073/04, de 28 de setembro de 2004, que instituiu tal programa, divide-o em atenção básica, média e alta complexidade para melhor organização do fluxo de pacientes, bem como sua distribuição na rede. Tal portaria assegura o acesso do cidadão ao Programa de Saúde Auditiva, que tem por objetivo desenvolver ações de promoção, prevenção, intervenção e desenvolvimento de atenção à saúde auditiva $^{17,18}$.

A atenção ao recém-nascido é realizada através da identificação precoce, por meio de triagem, de uma possível perda auditiva. Para que o diagnóstico seja concluído, os RN que "não passarem" na triagem devem ser referenciados para avaliação especializada. Esse encaminhamento também deve ocorrer sempre que seja identificada na história clínica presença de agravos que comprometam a saúde auditiva, e dos indicadores de risco para a deficiência auditiva segundo o Joint Committe of Infant Hearing e a recomendação do Comitê Brasileiro sobre Perdas Auditivas na Infância. Crianças com indicadores de risco para Deficiência Auditiva devem ser acompanhadas semestralmente até os três anos de vida ${ }^{8,19,20}$. Este estudo foi realizado com o objetivo de conhecer melhor a assistência e o cuidado à saúde auditiva dos RN nascidos nas maternidades da Rede Municipal de Saúde do Rio de Janeiro.

\section{Método}

Este foi um estudo observacional, descritivo e transversal, que teve como campo de pesquisa as Maternidades da Secretaria Municipal de Saúde do Rio de Janeiro que realizavam TAN. O projeto do estudo foi aprovado pelo Comitê de Ética em Pesquisa da Secretaria Municipal de Saúde do Rio de Janeiro em Julho de 2010. Para participação no estudo foi assinado o Termo de Consenti- 
mento Livre e Esclarecido por parte dos responsáveis pelas instituições e pelos responsáveis técnicos do serviço de Triagem Auditiva Neonatal. Os pesquisadores assinaram o Termo de Compromisso de utilização dos dados para acesso às informações dos prontuários. Foram incluídas neste estudo todas as maternidades sob gestão da Secretaria Municipal de Saúde do Rio de Janeiro que realizavam triagem auditiva neonatal e que concordaram com a participação e todos os $\mathrm{RN}$ vivos, nascidos nestas maternidades nos meses de outubro e novembro de 2010. Pelo montante de nascimentos e pelo fato de não haver grande diferença no funcionamento das maternidades durante os meses que antecederam o estudo, escolhemos somente dois meses para coleta de dados. A escolha do período se deu ao acaso.

O estudo foi realizado em duas etapas: a) Entrevista com os responsáveis pelo serviço de triagem auditiva em cada instituição participante para preenchimento do protocolo de observação e descrição do serviço; e, b) Coleta de dados em prontuários para observação dos indicadores de risco para Deficiência Auditiva na população nascida no período do estudo.

As variáveis estudadas foram: as maternidades da Secretaria Municipal de Saúde-RJ que realizam TAN, Recursos Humanos, Recursos Tecnológicos, número de RN que realizaram TAN e de RN que falharam na TAN, Indicadores de risco para Deficiência Auditiva e número de RN que foram atendidos pelo nível terciário.

Os dados foram coletados pela pesquisadora e por alunos treinados, pertencentes ao Programa de Iniciação Científica da Universidade Federal do Rio de Janeiro (UFRJ) por meio de formulários padronizados contendo perguntas fechadas para os responsáveis e protocolos de observação e documentos dos referidos centros: prontuários das maternidades, lista nominal do Programa de Atenção à Saúde Auditiva no Hospital Universitário Clementino Fraga Filho (HUCFF) da UFRJ. A Análise foi descritiva, com distribuição de frequências. O estudo foi realizado em três etapas: a) Entrevista com os responsáveis pelo serviço de triagem auditiva em cada instituição participante para preenchimento do Protocolo de observação e descrição do Serviço; b) Coleta de dados em prontuários para observação dos indicadores de risco para Deficiência Auditiva na população nascida no período do estudo; c) Coleta da lista nominal dos recém-nascidos vivos que chegaram ao Programa de Atenção à Saúde Auditiva HUCFF-UFRJ nascidos no período de Outubro e Novembro de 2010.

\section{Resultados}

Relacionamos seis maternidades sob gestão da Secretaria de saúde do município do Rio de Janeiro. Destas, foram identificadas três maternidades que realizavam triagem auditiva neonatal, que aceitaram participar do estudo e foram denominadas de A, B e C. A maternidade A apresentava registro de teste e reteste da TAN. A maternidade $\mathrm{B}$, como realizava TAN em sua maioria antes da alta hospitalar, só registrava o resultado final, mesmo que o teste fosse repetido para observar a confirmação ou não do resultado, no caso de falha. Na maternidade $\mathrm{C}$ foi possível registrar apenas o quantitativo de testes realizados, pois não havia registro de passa-falha na unidade, ou seja, o quantitativo de recém-nascidos que passaram ou falharam no teste da orelhinha, e os exames eram registrados na caderneta de vacinação. Foi identificado que todas as maternidades estudadas apresentavam fonoaudiólogos em seu quadro de funcionários com mesmo regime de trabalho e carga horária, além de estarem na função de "rotina" dos serviços.

Com relação aos recursos tecnológicos identificou-se que todas as unidades tinham aparelho da marca Maico-EROSCAM que realizava teste de Emissões Otoacústicas produto de distorção (EOA-PD). Além da realização de EOA-PD, as maternidades também realizavam a pesquisa do reflexo cócleo-palpebral.

A triagem auditiva na maternidade A era realizada preferencialmente em recém-nascidos de risco. Os sem risco eram orientados para retornar após a alta hospitalar. A maternidade B realizava triagem em todos os RN antes da alta hospitalar, visto que a triagem funcionava diariamente. Já a maternidade $\mathrm{C}$, realizava o serviço através de agendamento após a alta hospitalar e oferecia para todos os recém-nascidos da unidade.

Em todas as três maternidades os RN de baixo risco que passaram na triagem recebem alta com orientação sobre o desenvolvimento de linguagem e audição. Para os RN de baixo risco que falharam, os profissionais da maternidade A encaminham para avaliação no serviço de alta complexidade e outros locais; os das maternidades B e C encaminham para acompanhamento no serviço de follow up da unidade até aproximadamente o terceiro mês de vida. Nas três maternidades, os RN de risco que passaram na TAN são encaminhados para acompanhamento no serviço de follow up das unidades, que orientam sobre o desenvolvimento de audição e linguagem. Os RN de risco que falharam são encaminhados 
para o serviço da alta complexidade através do SISREG (sistema de agendamento e regulação de vagas do estado do Rio de Janeiro) e para outros locais e instituições para avaliação e diagnóstico em Deficiência Auditiva.

A alta complexidade é responsável pela avaliação, diagnóstico, intervenção (adaptação de prótese auditiva e indicação de implante coclear), reabilitação para crianças de até quatro anos de idade, crianças com múltipla deficiência ou afecções associadas à deficiência auditiva e daqueles que apresentarem dificuldades na realização da avaliação audiológica no serviço de menor complexidade. Além de ser responsável pela capacitação e treinamento dos demais níveis de comple$x^{x i d a d e}{ }^{18}$, o serviço de alta complexidade não recebeu nenhum dos recém-nascidos que falharam na triagem auditiva o que nos faz refletir sobre os atendimentos, uma vez que a triagem auditiva só se torna indispensável se os recém-nascidos forem atendidos pelo serviço de diagnóstico e intervenção.

No período do estudo foram identificados 3.824 RN vivos no Município do Rio de Janeiro. Nas maternidades que realizavam TAN, 1.865 RN vivos foram registrados. Destes, o total que realizou TAN foi de 757 (757/1.865; 40,5\%), mas se levarmos em conta o total de nascidos vivos identificados, o percentual de realização de TAN cai para 19,8\% (757/3.824). A Tabela 1 mostra a distribuição dos recém-nascidos vivos nas três maternidades que realizavam TAN.

A identificação dos recém-nascidos que falharam na TAN ocorreu apenas nas maternidades A e B, como mostra a Figura 1, uma vez que não há registro de passa-falha da maternidade C. Dos 1.079 registros de nascidos vivos nas maternidades A e B, 589 (54,6\%) realizaram TAN e destes, $573(97,3 \%)$ passaram na TAN e apenas $11(1,8 \%)$ falharam e precisariam ser encaminhados para avaliação e diagnóstico no serviço de alta complexidade.
Diante das porcentagens de cada maternidade, foi realizada análise individualizada de cada maternidade, como pode ser visto nas Figuras 2 e 3.

$\mathrm{Na}$ Figura 2 observa-se que após a realização do reteste, apenas oito $\mathrm{RN}$ falharam.

No entanto, é importante ressaltar que no mês de outubro a maternidade $B$ realizou triagem auditiva em 202/209 96,6\% dos nascidos vivos, e em novembro realizou apenas em 160/204 78,4\% devido às férias de uma das fonoaudiólogas. Isto foi significante para diminuir a capacidade de triagem auditiva nos resultados gerais. Estes valores indicaram que a maternidade $\mathrm{B}$, com todas as fonoaudiólogas atendendo no serviço, tem capacidade de triagem auditiva em quase $100 \%$ dos $\mathrm{RN}$ da unidade.

Identificamos que dos $11 \mathrm{RN}$ que falharam, oito $(72,7 \%)$ não tinham indicador de risco para surdez e dois $(18,2 \%)$ apresentavam pelo menos um indicador de risco, que foram: permanência em UTIN e uso de ventilação mecânica por mais de $48 \mathrm{~h}$, uso de medicamento ototóxico. O outro

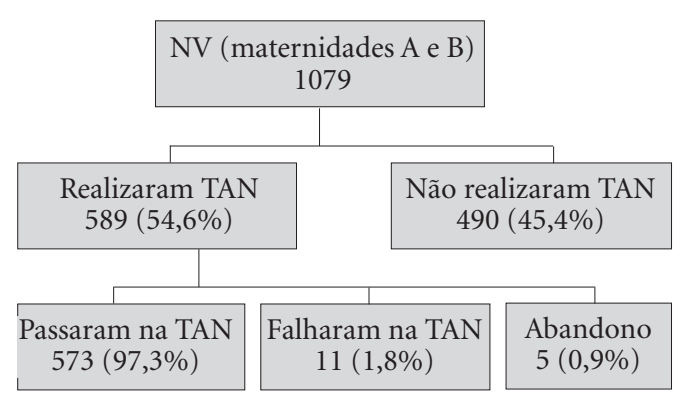

Figura 1. Fluxograma dos nascidos vivos segundo o passa-falha da Triagem Auditiva Neonatal nas maternidades A e B.

Nota: $\mathrm{NV}=$ nascidos vivos; $\mathrm{TAN}=$ Triagem Auditiva Neonatal.

Tabela 1. Distribuição dos recém-nascidos nas maternidades de estudo, de acordo com a realização da Triagem Auditiva Neonatal.

\begin{tabular}{lccc}
\hline & RN que realizaram TAN & RN que não realizaram TAN & Total de RN nascidos vivos \\
\hline Maternidade A & $227(34,1 \%)$ & $439(65,9 \%)$ & $666(100,0 \%)$ \\
Maternidade B & $362(87,6 \%)$ & $51(12,4 \%)$ & $413(100,0 \%)$ \\
Maternidade C & $168(21,4 \%)$ & $618(78,6 \%)$ & $786(100,0 \%)$ \\
Total & $\mathbf{7 5 7 ( 4 0 , 5 \% )}$ & $\mathbf{1 . 1 0 8}(\mathbf{5 9 , 5 \% )}$ & $\mathbf{1 . 8 6 5 ( 1 0 0 \% )}$ \\
\hline
\end{tabular}

Nota: $\mathrm{RN}=$ recém-nascidos; TAN = Triagem Auditiva Neonatal. 


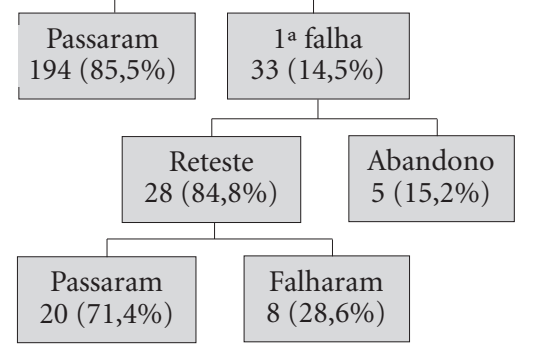

Figura 2. Fluxograma dos nascidos vivos na maternidade A e a distribuição segundo o passa-falha da Triagem Auditiva Neonatal.

Nota: $\mathrm{NV}=$ nascidos vivos; RTAN $=$ realizaram Triagem Auditiva Neonatal; NRTAN = não realizaram Triagem Auditiva Neonatal.

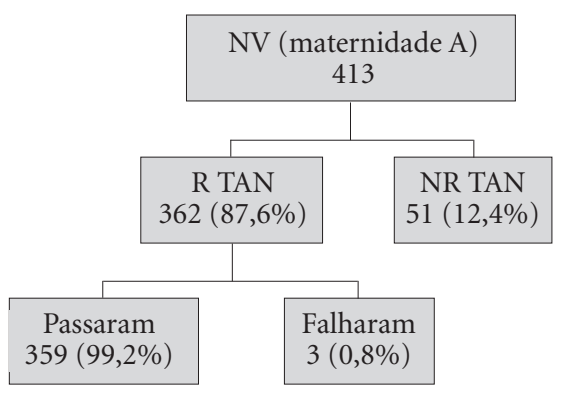

Figura 3. Fluxograma geral dos nascidos vivos na maternidade B e sua distribuição segundo passa-falha da Triagem Auditiva Neonatal.

Nota: $\mathrm{NV}=$ nascidos vivos; $\mathrm{RTAN}=$ realizaram Triagem Auditiva Neonatal; NRTAN = não realizaram Triagem Auditiva Neonatal.

bebê não teve seu prontuário localizado dentro da maternidade.

Observamos que dos 11 recém-nascidos que falharam na triagem auditiva nas maternidades $\mathrm{A}$ e B, nenhum chegou para avaliação, diagnóstico auditivo e intervenção. Não foi possível localizar esses $\mathrm{RN}$ que falharam devido à incompatibilidade de dados entre as maternidades e o HUCFF -UFRJ. Através da lista do Serviço Social e do Serviço de Fonoaudiologia no centro de alta complexidade não se encontrou registro de crianças menores de dois anos, na época do estudo.

\section{Discussão}

Este estudo foi pioneiro no que diz respeito ao olhar sobre a rede de Atenção à Saúde Auditiva Neonatal no Município do Rio de Janeiro e verificou que as três unidades materno-infantis escolhidas para o estudo realizavam triagem auditiva neonatal, apesar de não conseguirem atender toda a demanda. Nossos resultados apontaram para 1,8\% de falha na Triagem Auditiva Neonatal, o que se encontra de acordo com a orientação do Comitê Brasileiro de Perdas Auditivas na Infância ${ }^{21}$, em que é sugerido percentual de encaminhamento para avaliação audiológica completa pós-triagem de no máximo $4 \%$. Por outro lado, $80,2 \%$ dos nascidos vivos nas maternidades municipais do Rio de Janeiro, no período do estudo, não realizaram triagem auditiva neonatal, revelando um percentual baixo de realização da TAN, aquém do referido na literatura nacional${ }^{22-24}$ e indicado pelo Comitê Multiprofissional em Saúde Auditiva ${ }^{25}$, que considera um serviço de triagem eficaz quando ele atende pelo menos $95 \%$ dos recém-nascidos vivos. Entretanto, ao considerarmos uma das maternidades (maternidade B), verificamos que o percentual mostrouse adequado, assim como em uma maternidade de São Paulo, na qual foram avaliados $94,8 \%$ dos $\mathrm{RN}$ em um período de cinco meses ${ }^{22}$. Em uma maternidade localizada em Santa Catarina foram avaliados $81,7 \%$ dos $\mathrm{RN}^{23}$ e no Espírito Santo, em duas maternidades públicas, foram avaliados $67,9 \%$ dos RN em um período de três anos ${ }^{26}$. De acordo com a Lei Municipal 3028/2000, todos os nascidos vivos no Município do Rio de Janeiro devem ser triados imediatamente após o nascimento, uma vez que a perda auditiva permanente necessita de intervenção nos primeiros anos de vida para apresentar bons resultados no processo de reabilitação da audição e da linguagem ${ }^{27}$.

Com relação aos indicadores de risco para perdas auditivas, observamos uma prevalência de permanência em UTIN por mais de $48 \mathrm{~h}$, seguido do uso de medicamentos ototóxicos, ventilação mecânica e índice de apgar de 0 a 4 no $1^{\circ}$ minuto ou de 0 a 6 no $5^{\circ}$ minuto. Estes indicadores de risco geralmente não acontecem de forma isola- 
da e podem ser minimizados com melhor assistência perinatal que envolve atenção e cuidado a gestante desde a consulta pré-natal. A redução no número de RN de risco influencia diretamente na assistência e cuidado aos RN que precisam de acompanhamento posterior, uma vez que os serviços reduziriam suas filas de espera e otimizariam as avaliações desses RN. Além de diminuir a demanda para atendimento, reduziriam os gastos com cuidados perinatais e acompanhamento audiológico dessas crianças. Dentre os RN que falharam na TAN, encontramos dois com indicadores de risco para perda auditiva; Barreira et al. ${ }^{25}$ encontraram $12,5 \%$ dos RN triados com indicadores de risco para perda auditiva.

No que se refere aos critérios técnicos e de qualidade de serviço, podemos observar que das maternidades que realizavam TAN, apenas a $\mathrm{B}$ conseguiu atendê-los. Entretanto, não foi possível observar os índices de falso positivo e negativo porque não houve conclusão diagnóstica dentro do Programa de Atenção à Saúde Audi- tiva. Podemos inferir que a situação da TAN nas maternidades municipais do Rio de Janeiro precisa de ajustes para melhor contribuir com a saúde pública.

Algumas limitações permearam o estudo e dentre elas, podemos citar que, dos 757 nascidos vivos que realizaram TAN, $77,81 \%$ apresentavam registro em prontuário; os demais possuíam anotação em carteira de vacinação, o que dificultou o resgate das informações. Não foi possível constatar a conclusão diagnóstica dentro do Programa de Atenção à Saúde Auditiva em função da inexistência dos dados de chegada dos $\mathrm{RN}$ no nível de alta complexidade. A Triagem Auditiva Neonatal é apenas o início do Programa de Atenção a Saúde Auditiva do recém-nascido. Sendo assim, é fundamental que os serviços de triagem funcionem integrados à rede de atenção à saúde auditiva através do Programa de Atenção à Saúde Auditiva do Governo Federal que prevê avaliação, diagnóstico, intervenção e reabilitação adequadas ao recém-nascido ${ }^{27}$.

\section{Colaboradores}

PT Lima, MG Goldbach, MC Monteiro e MG Ribeiro participaram igualmente de todas as etapas de elaboração do artigo.

\section{Agradecimentos}

Às alunas do Programa de Iniciação Científica do Curso de Fonoaudiologia da UFRJ, que estiveram de frente a todo esse trabalho em campo: Danielle, Flavia, Nasila, Thiago, Juliana, Gabriella, Edilaine, Bianca, Camila, Thalyta, Raquel e Tamires e as fonoaudiólogas das unidades materno-infantis que nos receberam muito bem e contribuíram de forma importante para este trabalho. 


\section{Referências}

1. American Academy of Pediatrics (AAP). Position Statement, 1992 - Joint Committee on Infant Hearing. Pediatrics 1992; 70:496-497.

2. American Academy of Pediatrics (AAP). Task force on Newborn and Infant hearing. Newborn and Infant Hearing Loss: Detection and Intervention. Pediatrics 1999; 103(2):527-530.

3. Basseto MCA. Triagem Auditivas em Neonatos. In: Campiotto AR, Levy C, Redondo MC, Anelli W, Lopes Filho O, organizadores. Tratado de Fonoaudiologia. Ribeirão Preto: Tecmedd; 2005. p. 223-233.

4. Pádua FGM, Marone S, Bento RF, Carvallo RMM, Durante AS, Soares JC, Barros JCR, Leoni CR. Triagem Auditiva neonatal: um desafio para sua implantação. Rev Bras Otorrinolaringol 2005; 9(3):189-194.

5. Yoshinaga-Itano, Sedey AL, Coulter DK, Mehl AL. Language of Early- and Later-identified Children With Hearing Loss. Pediatrics 1998; 102(5):1161-1171.

6. Yoshinaga-Itano C. Early identification: an opportunity and challenge for audiology. Semin Hear 1999; 20(4):317-331.

7. Kuhl PK, Willians KA, Lacerda F, Stephens KN, Lindbloom B. Linguistic experience alters phonetics perception in infants by six month of age. Science 1992; 255(5044):606-608.

8. Comitê Brasileiro sobre Perdas Auditiva na Infância (CBPAI). $1^{\circ}$ Recomendação: período neonatal. J Pediatr 2001; 77-81.

9. Colorado Newborn Hearing Screening Project. American Academy of Audiology Annual Convention. Chicago, Illinois: 2000. [acessado 2003 jul 15]. Disponível em: http://www.colorado.edu/slhs/mdnc

10. Câmara Municipal do Rio de Janeiro. Lei Municipal no 3028, de 17 de Maio de 2000. Diário Oficial do Rio 2000; 18 maio.

11. Brasil. Ministério da Saúde (MS). Lei Federal no 12303 , de 2 de Agosto de 2010. Dispõe sobre a obrigatoriedade de realização do exame denominado Emissões Otoacústicas Evocadas. Diário Oficial da União 2010; 3 ago.

12. Kos MI, Frota S. Aplicações clinicas das Emissões Otoacústicas Evocadas Transientes e Produto de Distorção. In: Frota S, Goldfeld M, organizadoras. Ouvir e Falar Vol. 3 Enfoques em Audiologia e Surdez. São Paulo: AM3; 2006. p. 38-44.

13. Chapchap MJ, Segre CM. Universal newborn hearing screening and transient evoked otoacoustic emission: new concepts in Brazil. Scan Audiol Suppl 2000; (53):33-36.

14. Kemp DT, Ryan P, Brain PA. A guide to the effective use of otoacoustic emissions. Ear Hear 1990; 11(2):93-105.

15. CBPAI - Comitê Brasileiro sobre Perdas Auditivas na infância. Recomendação 01/99 do Comitê Brasileiro sobre perdas auditivas na infância. J Cons Fed Fonoaudiol 2000; 5:3-7.

16. Instituto Brasileiro de Geografia e Estatística (IBGE). Senso demográfico (2003), Características gerais da população. Rio de Janeiro: IBGE; 2003.
17. Brasil. Ministério da Saúde (MS). Portaria no 2073/ GM, de 28 de Setembro de 2004. Institui a Política Nacional de Atenção à Saúde Auditiva. Diário Oficial da União 2004; 29 set.

18. Brasil. Ministério da Saúde (MS). Portaria no 587, de 7 de Outubro de 2004. Diário Oficial da União 2004; 8 out.

19. Joint Committee on Infant Hearing Position Statement. Principles and Guidelines for early hearing detection and intervention programs. Am J Audiol 2000; 9(1):9-29.

20. Joint Committee on Infant Hearing 2007 Position Statement. Principles and guideslines for early hearing detection and intervention programs. Pediatrics 2007; 120(4):898-921.

21. Durante AS, Mamede RM, Costa MTZ, Cianciarullo A, Voegels RL, Takahashi GM, Soares AVN, Spir EG. A implementação de programa de triagem auditiva neonatal universal em um hospital universitário brasileiro. Rev. de Pediatria 2004; 26(2):78-84.

22. Mattos WM, Cardoso LF, Bissani C, Pinheiro MMC, Viveiros CM, Carreirão Filho W. Análise da implantação de programa de triagem auditiva neonatal em um hospital universitário. Braz J Otorhinolaryngol 2009; 75(2):237-244.

23. Fernandes JC, Nozawa MR. Estudo da efetividade de um programa de triagem auditiva neonatal universal. Cien Saude Colet 2010; 15(2):353-361.

24. Lewis DR, Marone SAM, Mendes BCA, Cruz OLM, Nóbrega M. COMUSA- Comitê de Programa de Saúde Auditiva em duas maternidades públicas. Rev Soc Bras Fonoaudiol 2007; 12(2):99-105.

25. Barreira C, Neto HAF, Gattaz G. Processo de implantação de Programa de Saúde Auditiva em duas maternidades públicas. Rev Soc Bras Fonoaudiol 2007; 12(2):99-105.

26. Yoshinaga-Itano C. From screening to early identification and intervention: Discovering predictors to successful outcomes for children with significant hearing loss. J Deaf Stud Deaf Educ 2003; 8(1):11-30.

27. Guimarães VC, Barbosa MA. Avaliação auditiva no recém-nascido e suas implicações éticas. Cien Saude Colet 2010; 15(2):559-562.

Artigo apresentado em 28/10/2013

Aprovado em 29/03/2014

Versão final apresentada em 08/04/2014 
